\title{
Wireless Sensor Networks for Structural Health Monitoring: A Multi-Scale Approach
}

\author{
Authors: \\ Tracy Kijewski-Correa, Dept. of Civil Engineering \& Geological Sciences, University of \\ Notre Dame, 156 Fitzpatrick Hall, Notre Dame, IN 46556, tkijewsk@nd.edu \\ Martin Haenggi, Dept. of Electrical Engineering, University of Notre Dame, 274 \\ Fitzpatrick Hall, Notre Dame, IN 46556, mhaenggi@nd.edu \\ Panos Antsaklis, Dept. of Electrical Engineering, University of Notre Dame, 273 \\ Fitzpatrick Hall, Notre Dame, IN 46556, pantsakl@nd.edu
}

\begin{abstract}
Given the present burdens associated with inspection and maintenance of Civil Infrastructure, the development of effective, automated damage diagnosis techniques, including the sensor technologies that support them, has become a major research need. While recent developments in wireless sensor networks have demonstrated their potential to provide continuous structural response data to quantitatively assess structural health, many important issues including network lifetime and stability, damage detection reliability, and trade-offs in model order to balance computational capabilities must be realistically addressed. Only then can wireless embedded sensor networks become a practical tool for Structural Health Monitoring of large, complex Civil Structures. In response to these needs, the concept of a multi-scale wireless sensor network is introduced in this study with a restricted input network activation scheme and the integration of data from a heterogeneous sensor array to improve damage detection for low-order models. The multi-scale network concept introduced here helps to improve power efficiency, minimize packet loss and latency, and eliminate synchronization issues through the use of a decentralized analysis scheme and the activation of sub-networks only in the vicinity of suspected damage, while reducing the required size of the reference pool for the undamaged state. This study introduces the network architecture concept, a strain-driven approach to damage detection and preliminary simulated results.
\end{abstract}

\section{Previous Work in Wireless Structural Health Monitoring}

Most research in these areas of "intelligent" structural assessment and evaluation retain the traditional architecture of a centralized data acquisition hub wired to tens or even hundreds of sensors. As cost effectiveness is a major concern, the installation and maintenance of these cabled systems represent significant concerns, prompting the move toward wireless sensor networks, particularly those with local processing capabilities, thus reducing the amount of data transmitted in power-consuming wireless radio communications. Early work in this area was led by Straser and Kiremidjian [1] and 
advanced by Lynch et al.'s [2] dual processor format, followed by a later generation lowpower, multi-channel prototype [3]. Another example is the multi-hop WISDEN system [4], which uses the small MICA2 Motes, developed at the University of California at Berkeley [5]. In all of these applications, by reducing transmission from lengthy time histories to a number of key parameters, battery life of the wireless nodes can be extended, while the issues of strict time synchronization and loss intolerance in harsh environmental conditions are marginalized. Building on these prior efforts, the proposed approach offers a multi-scale network topology that is inherently more scaleable and exploits short wired communications for better energy-efficiency and reduced latency.

\section{SUMMARY OF KEY FEATURES}

Though such efforts have certainly made important contributions to the field of structural health monitoring (SHM), the potential of wireless sensor network technologies has yet to be fully tapped. While researchers are devoting considerable effort to the issue of power scavenging, current wireless networks still rely on battery power - an admitted limitation. Thus, these networks must be power-efficient not only in their hardware and software, but also in their overall network architecture - yet another reason to fully exploit on-board computational capabilities. As such, this study builds upon the earlier efforts in [6], which utilized the capability for decentralized data analysis and a multiscale format, and introduces a strain-driven system identification approach and Restricted Input Network Activation Scheme (RINAS) to achieve several benefits:

- improved decentralized damage detection through the fusion of data from spatially distributed heterogeneous sensor arrays, using low-order models to reduce computational burden and extend network lifetime;

- reduction of reference pool required to identify damage from ambient vibration data, enhancing the reliability of detection while reducing computational requirements;

- additional improvements in power efficiency and network performance through the use of event triggering and a network architecture utilizing only sub-networks in the vicinity of damage.

Heterogeneous sensing is achieved in this study through the integration of strain gages and accelerometers. It is demonstrated that this fusion of acceleration and strain intercepts damage in earlier stages than the use of accelerometer data alone [7]. From the networked sensing perspective, this effort represents a significant deviation from the traditional approach to wireless networks by tailoring an application-specific network and activation scheme that is partnered with a decentralized system identification approach that is itself network-specific and computationally efficient. As such, this study introduces a number of innovations in embedded sensor networks for practical and effective damage detection Civil Infrastructure Systems (CIS).

In order to achieve the concept of a multi-scale wireless sensor network for SHM, this study presents the following specific objectives (O1-O4), which will be detailed in subsequent sections with preliminary results to demonstrate proof-of-concept:

- O1. Design a power-efficient multi-scale network architecture to enhance damage detection capabilities through spatial cross-validation.

- O2. Develop a damage detection approach, which fuses data from a heterogeneous, spatially distributed sensor array, and a restricted input network activation scheme to 
enhance reliability of detection under ambient vibrations and reduce local computational burdens.

- O3. Adapt a commercially-available wireless hardware platform to accommodate heterogeneous sensing and execute the proposed system identification scheme in a power-efficient manner.

- O4. Prototype the system using integrated simulation based upon full-scale data sets, field testing and bench scale experimentation, followed by full-scale deployment.

\section{CONSTRAINTS \& OPERATIONAL REQUIREMENTS OF THIS APPLICATION}

Off-the-Shelf Hardware. Although there have been a number of proprietary wireless platforms designed, the authors will use commercially available platforms for a variety of reasons: (i) authors' established expertise with these platforms, (ii) commercial availability, which reduces cost and insures findings can be replicated and expanded upon by other researchers, and (iii) power efficiency of the platform design. The choice of sensors involved in the hardware design also minimizes cost, so that a dense sensor array can be reasonably achieved.

Minimize Power Consumption. Network architectures and system identification approaches introduced in this study seek explicitly to minimize power consumption to extend the life of the network. As such, a number of trade-offs in the model orders used in the computational scheme and the sensors selected are inevitable.

Pseudo-Real Time Responsiveness. While the system must be capable of rapidly delivering an assessment of the bridge to the end user, this need not take place in a strictly real-time fashion. Latency of a few minutes from the measurement of response to the notification of the end user is completely reasonable for this application, as structural failures in these systems will not be immediate. As such, computational and communications resources within the network need not be extensive, as any limited resources can be allocated accordingly over a number of minutes.

Reliance on Ambient Excitations. While it is acknowledged that controlled excitations simplify the system identification aspects by strictly regulating the input and exciting the higher modes more sensitive to damage, their implementation is not practical for many in-service structures due to disruption of traffic, cost, and intrusion. Thus it is highly desirable to develop a diagnostic strategy that is reliant upon naturally occurring or ambient excitations, e.g., traffic. In this study, though ambient vibration data is used, the inputs to the system are effectively controlled by RINAS, which minimizes the possible environmental and operational states. Meanwhile, the use of a heterogeneous sensing alternative is presented to compensate for the sole use of lower mode responses, as higher mode effects are not envisioned to contribute significantly.

\section{ObJective 1: The NeTwork ConcePt AND ArChitecture}

\section{Network Concept}

The overall multi-scale network concept, introduced first in [6], is generally demonstrated on the simply supported beam shown in Figure 1. The structure is first subdivided into zones to aid in the localization of damage. Each zone contains sensors capable of detecting two "scales" of response: accelerations (MESO- or m-nodes) and 


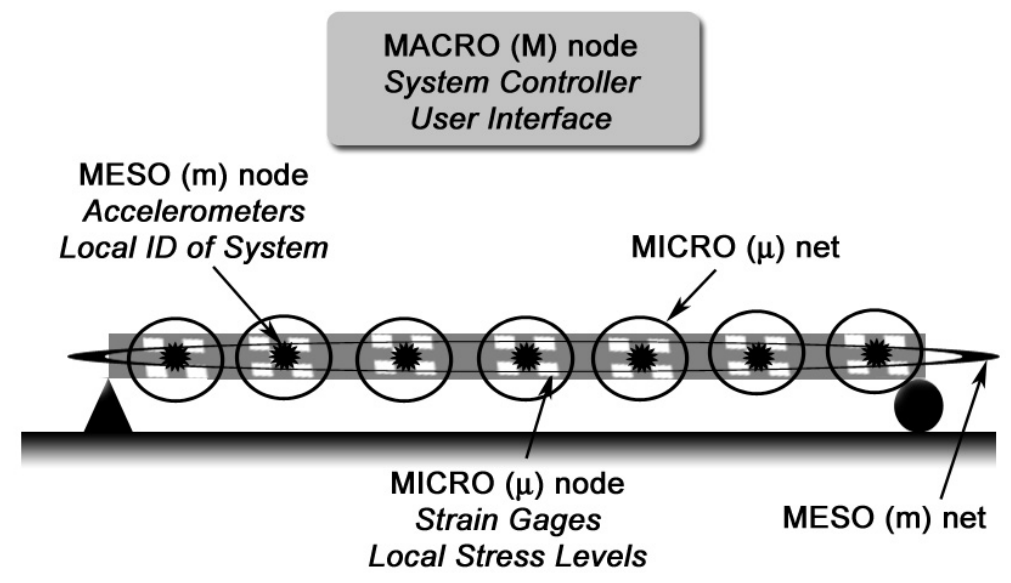

FIGURE 1

SCHEMATIC OF MULTI-SCALE NETWORK CONCEPT ON SIMPLE BEAM

strain gages (MICRO- or $\mu$-nodes) that provide a highly localized indication of stress concentration/strain behavior at critical locations, including the underside of the deck, joints, transverse beams near the supports, braces/ribs, and near upper flanges of girders likely to corrode. Within each zone, accelerometers and strain gages share information across scales to aid in the damage detection process, forming a local network (MICROor $\mu$-net). Meanwhile, accelerometers also share information across zones by interfacing with neighboring accelerometers to form a larger network (MESO- or m-net) to enhance reliability. All activities of the network are triggered by a central node (MACRO- or Mnode) monitoring the approaching traffic conditions through a system of cameras. This information is then used by the M-node to trigger the network of bridge response sensors according to the aforementioned RINAS scheme (discussed in more detail in a subsequent section). This M-node also receives information on damage states wirelessly from the network and interfaces with the end user via an off-site web server to report these findings or to permit a manual trigger of the system. Connectivity between the offsite web server and the M-node can be achieved via Ethernet or a cellular link.

While each node of the $\mathrm{m}$ - and $\mu$-nets should ideally be untethered, using wireless communications and on-board batteries, the design presented here offers some compromise. The $\mu$-nodes are wired locally to a m-node, forming a $\mu$-web to locally concentrate power and processing. As such the m-node forms a datalogger-like hub [8], which can also interface with a variety of sensors. Note that this represents a first approach to the multi-scale network concept, and future designs would have a completely wireless approach at all scales.

Communications within the network are designed to maximize battery life at each of the m-nodes. The decentralized system identification approach and the utilization of $\mu$ webs aid in this goal by minimizing the amount of data transmitted wirelessly, and thereby the power depleted. However, the very architecture of the network is also designed with power conservation in mind. The network is only activated by RINAS on a pre-defined schedule, e.g., once per week or month, when desired traffic conditions are observed. All nodes in the m-and $\mu$-nets remain dormant until a RINAS activation order or end-user request is received from the M-node. As shown in Figure 2, the detection 


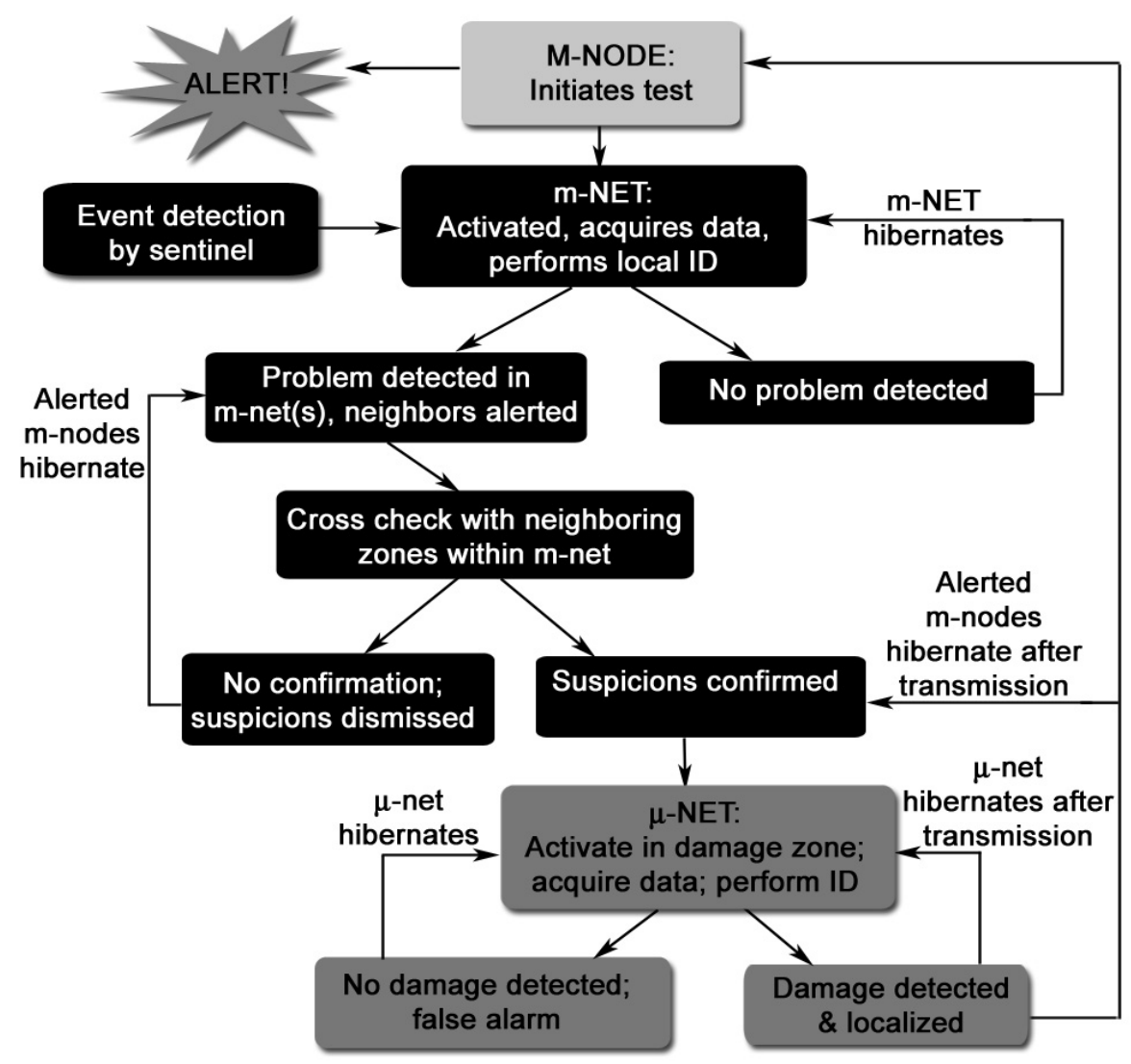

\section{FIGURE 2}

DECISION TREE FOR MULTI-SCALE NETWORK CONCEPT

process begins with the activation of all nodes in the m-net. The m-nodes collect time histories and perform system identification locally, allowing a determination of potential damage. Nodes detecting damage remain alert and notify their nearest neighboring mnodes so that findings may be compared within the m-net to help validate the authenticity of the damage suspicion. In this process, all nodes that do not participate in such local collaboration return to a hibernation state to conserve power. If damage is indeed confirmed within the m-net, the $\mu$-net associated with the zone of damage is activated to begin taking strain data in parallel with the accelerations detected at the m-node, and this data is fused in a decentralized system identification approach to either corroborate or dismiss the initial damage claim. If the damage claim cannot be validated by the m-node, the $\mu$-and m-systems return to a hibernating state. If the damage claim is validated by the $\mu$-net, its location within the $\mu$-net is estimated and then reported back to the M-node through a multi-hop communication scheme. As such, the role of the sensor network is extended from simply a means for communication to a technology that can be engineered to improve structural assessment through the interaction of sub-networks.

\section{Network Architecture}

The goal of this task is to investigate and design reliable and energy-efficient protocols for data acquisition, processing, and transmission that are tailored to the SHM application 
and operate under various bounds such as maximum latency and minimum lifetime. From a theoretical point of view, the authors aim at a sound analysis of the system performance, whereas the experimental part will be used for verification purposes. While the architecture used for the experimental part of this project is intentionally kept simple, the scope of this research thrust goes beyond the architecture shown in Figures 1 and 2 and will be a firm base for larger and more complex networks that extend in multiple dimensions, consist of heterogeneous nodes, and encompass several hierarchical levels.

Numerous issues need to be addressed in the implementation of a sensor network. For some of them, standard solutions found in the literature or derived from the authors' own experience in the their DARPA/IXO NEST (Networked Embedded Systems Technology) project are applicable; others require further investigation and study. For the SHM application, there are mainly two parts of the network protocol stack where fundamental research is needed: channel access and data acquisition.

The Wireless Channel. Many wireless solutions have been proposed for SHM [1, 2, 4] but virtually all of them consider the wireless connection merely as a convenient replacement for wires that would be expensive to deploy. The stochastic nature of the wireless channel is neither explicitly considered nor exploited, which leads to inefficient protocol and overall system design.

In this approach, the wireless channel will be taken into account explicitly by (1) characterizing its properties in detail (including, e.g., small-scale fading) and (2) exploiting its properties whenever possible (e.g., the broadcast nature that permits local data distribution at no additional energy expenditure). In the presence of (static) smallscale fading, physical proximity does not guarantee good links, and, in turn, relatively long links may still exhibit small path loss. With pedestrian or vehicular traffic on a bridge, the channel is also subject to dynamic small-scale fading and possibly shadowing. Such relatively slow fluctuations in the channel quality are problematic due to the lack of time diversity; thus the network needs to adapt and self-configure to such slow changes in the link quality. Opportunistic approaches are promising and their feasibility will be explored in this project; however, they also raise fairness and latency concerns, and they often require knowledge of the channel state that may be difficult to obtain. These tradeoffs will be studied in the context of the given application, extending prior work but with substantially more realistic models and backed up by experimentation.

Channel Access. In any multi-node or multi-user wireless system, a MAC (multipleaccess) layer is needed that regulates the channel access. Channel access is an instance of a distributed resource allocation problem that includes space, time, bandwidth, and possibly power. In an energy-constrained network with event-driven traffic, this is a particularly critical issue, since many nodes will start sensing and transmitting simultaneously when an event occurs. The MAC scheme needs to be specifically designed for these phases of high traffic. While continuous traffic flows can be accommodated by reservation-based schemes (TDMA, FDMA, CDMA), such schemes with fixed assignment do not provide the flexibility called for by this application. Random access techniques with smart adaptive backoff or a negotiation or contention phase are more promising, but standard solutions such as the CSMA-based MAC scheme in the IEEE 802.11 standard perform suboptimally. Cleary, an application-specific solution is called for. 
If the M-node is able to reach all the lower-tier nodes, it is possible to use centralized scheduling schemes where the M-node assigns time slots or polls the m-nodes. In addition, the M-node could ensure tight synchronization among the nodes, which helps scheduling transmissions and sleep intervals. For the upstream link, however, some mnodes will most likely be unable to reach the M-node directly, so they have to find multihop routes and use other nodes as relays to deliver their data. Since the network topology is fixed, routing is not the primary concern. There is, however, an interdependence between routing and channel access. The spatial correlation of the data needs to be exploited at the MAC layer in the same way this has been done at the network layer. Since neighboring nodes are likely to report similar data, the traffic load can be reduced accordingly.

Data Acquisition and Local Processing. Significant energy and reliability gains can be expected from collaborative data acquisition and local preprocessing, since nearby $\mu$ and m-nodes will sense correlated data and short-distance communication is inexpensive and robust. It is therefore sensible to investigate distributed detection and compression schemes at the local level ( $\mu$ - and m-nodes).

A key idea that will be pursued is based on the fact that temporal and spatial sampling can be traded off. Tight synchronization permits tradeoffs between temporal and spatial sampling. High temporal resolution is achieved if the nodes' sampling rates are identical but the sampling times have a relative offset. High spatial resolution is achieved when all nodes sample at the same time instants. So, depending on the size of a phenomenon, the application can ask for high temporal or spatial resolution, or the m-nodes themselves can switch to higher temporal resolution if the sensed data within their clusters is very similar. Within the same framework, the quantization can be dynamically adjusted. An information-theoretic rate-distortion measure can be used to assess the performance of the end-to-end communication system.

Note that the architectural and networking issues are not independent and thus cannot be addressed in isolation. Rather, a so-called cross-layer (or "holistic") approach will be adopted, which is distinct from previous work in SHM. In particular, both the channel access scheme and the local processing algorithms need to be tied in with the network structure described in the previous section: the MAC (and routing) layer needs to be aware which nodes are awake, and for local processing and data exchange, nodes need to be woken up.

\section{Objective 2: Damage Detection by Heterogeneous Data Fusion}

The Civil and Mechanical Engineering communities have invested significant efforts toward damage detection, as summarized in a number of literature reviews [9]. Though many of these approaches were developed for integrity assessment of mechanical components and aerospace structures, they have recently been translated into larger Civil Engineering Systems. These approaches typically rely on an aggregation of time histories for a centralized approach to system identification. As such, they have not been developed to maximize the full potential of wireless embedded sensor networks with local processing capabilities. For this reason, fundamental research in decentralized system identification is necessary and has been undertaken in some early work in wireless sensor networks for SHM. These approaches focused on the frequency [10] and/or time domains [11]. In either case, the success of damage detection was enhanced 
by decentralized system identification, where denser sensor arrays offered a more localized sensitivity to changes in structural properties.

However, the research to date has not considered the necessary trade-offs in model orders to ease computational burdens. This study responds to this need by investigating the potential compensation offered by the fusion of data from heterogeneous, spatially distributed dense sensor arrays in the context of a decentralized time domain approach using ambient vibration. The motivation for the use of a heterogeneous array is based on the work by Law et al. [7], which demonstrated that the combined use of strain data and accelerometer data outperformed the sole use of acceleration data in damage detection in a wavelet packet approach. This study builds upon this notion and bases its system identification approach on the two-stage prediction model first proposed by Sohn and Farrar [11], referred to herein as the LANL (Los Alamos National Laboratories) scheme, rooted in statistical pattern recognition and autoregressive/autoregressive with exogenous inputs (AR/ARX) modeling.

In the LANL scheme, inputs to the system are generically assumed to be white noise, through the ARX modeling. In the modified scheme presented here, damage detection will be achieved similarly through time series analysis, though regressively modeling the relationship between strain and acceleration, instead of a random white noise input. A summary of the scheme is now presented:

1. A reference pool of normalized undamaged acceleration time histories $a_{x}$, under various operational and environmental conditions, is modeled by an AR representation:

$$
a_{x}(t)=\sum_{j=1}^{p} \phi_{x j} a_{x}(t-j)+e_{x}(t)
$$

where $t$ is a discrete time index, $\phi_{x j}$ is the AR coefficient for the time series $a_{x}, e_{x}$ is the random error term, $p$ is the order of the AR model and $j$ is an integer index.

2. An acceleration response time history $a_{y}$, under an unknown condition, is collected, normalized and fit:

$$
a_{y}(t)=\sum_{j=1}^{p} \phi_{y j} a_{y}(t-j)+e_{y}(t)
$$

where $\phi_{y j}$ is the AR coefficient for the time series $a_{y}$ and $e_{y}$ is the random error term.

3. The reference pool from step 1 is searched to determine the reference state best matching $a_{y}(t)$. Note that identification of the reference state uses only the acceleration data to reduce computational burden.

4. In the original LANL scheme, the residual errors $e_{x}$ and $e_{y}$ are assumed to be the result of the external input to the system treated as white noise and are thus used to drive an ARX model. It is proposed here, instead of treating these errors as inputs, to regressively model the simultaneously-acquired strain $s_{x}$ and acceleration $a_{x}$ data, which will be observed for changes associated with damage of the structure through an autoregressive moving averages (ARMA) modeling, with a residual error of $\varepsilon_{x}(t)$ :

$$
a_{x}(t)=\sum_{i=1}^{a} \alpha_{i} a_{x}(t-i)+\sum_{j=1}^{b} \beta_{j} s_{x}(t-j)+\varepsilon_{x}(t)
$$

where $\alpha_{i}$ and $\beta_{j}$ are the coefficients of the ARMA model, $a$ and $b$ are the orders of the ARMA model, and $i$ is an integer index. 
5. This same ARMA model is then applied to $a_{y}(t)$ to determine the accuracy of the representation:

$$
\varepsilon_{y}(t)=a_{y}(t)-\sum_{i=1}^{a} \alpha_{i} a_{y}(t-i)-\sum_{j=1}^{b} \beta_{j} s_{y}(t-j)
$$

where $\varepsilon_{y}$ is the residual error on the fit.

6. A ratio of the standard deviations of the residual errors $\left(\sigma\left(\varepsilon_{y}\right) / \sigma\left(\varepsilon_{x}\right)\right)$, termed $h$, is then defined as the damage metric and is expected to take on its largest values at nodes closest to the damage location. In order to distinguish meaningful damage from inherent variability, a statistical significance threshold is defined by applying the ARMA model in step 4 to the remaining records in the reference pool to determine the probability distribution on $h$ and define an appropriate upper confidence limit (95\% to 99\%) for undamaged states. Damage is suspected if the damage metric $h$ for the record of interest surpasses this threshold. Note that in many statistical pattern recognition applications, these distributions on the damage metric are assumed Gaussian [11], though this may increase the number of false positives if the distribution is actually long-tailed, warranting the use of extreme value statistics [12].

Though larger model orders $(p=30)$ have been used in the literature [11, 8], this requires very expensive computational resources. The proposed strain-driven ARMA identification technique, which uses ambient vibrations and is thus not characterized by higher mode responses, permits the use of lower model orders without a loss of accuracy. This will be demonstrated in the following section for beam elements, while a later section will document that model orders between 5 and 15 can be successfully embedded in the proposed hardware without the need of writing to the flash memory, which is a costly operation in terms of power consumption.

Initial Proof of Concept. To demonstrate the benefits of the proposed strain-driven ARMA approach versus the original LANL scheme, a finite element model is used to simulate the acceleration and surface strain time histories at four equispaced points along the longitudinal dimension of a thin beam shown in Figure 3a. Only the first two modes are retained for the simulation. The beam is excited by ambient inputs modeled as random, Gaussian white noise. Damage introduced to the beam is comprised of a transverse cut, symmetrically imparted at midspan, specified as a percentage of the total transverse dimension of the beam, as shown in Figure 3b. As an important trade-off in damage detection capability and computational burden is necessary for feasible implementation on the wireless platform, the efficacy of low-order models is demonstrated herein ( $p=6, a=3, b=3$ ). The reference pool is defined by 300 undamaged data sets generated from independent, random excitations to the system, subsequently fit by the AR model in (1).

Various damage schemes, summarized in Table 1 , were simulated. The LANL scheme is applied only to the simulated acceleration data, while the newly proposed strain-driven ARMA approach is applied to the acceleration and surface strain. Subsequently, these two modeling approaches are used to conduct an analysis of the reference pool to establish the statistical significance threshold for the damage metric $h$, defined as the $97.5 \%$ threshold value for a normally-distributed variate. For the LANL scheme, this threshold is 1.31 and is 1.15 for the proposed strain-driven ARMA method. As demonstrated by the results in Table 1 , for detection at $\mathrm{L} / 2$, the proposed strain-driven ARMA method enables detection of even more subtle levels of damage than the LANL 
scheme, using relatively low model orders. While this example shows the promise of using heterogeneous sensor outputs, more work needs to be done to compensate for nonGaussian distributions of the damage metric and to document the performance of this approach on more complex systems and under other damage scenarios.

Note that for consistency with the proposed network scheme, strains are only acquired once damage is suspected through an analysis of acceleration data in the m-net. As such, the original LANL scheme is executed within the m-net and the proposed straindriven technique is subsequently executed in the $\mu$-net. To insure that potential damage states will not be disregarded at the m-net, the confidence limit percentile on the threshold value for the LANL scheme will be relaxed.
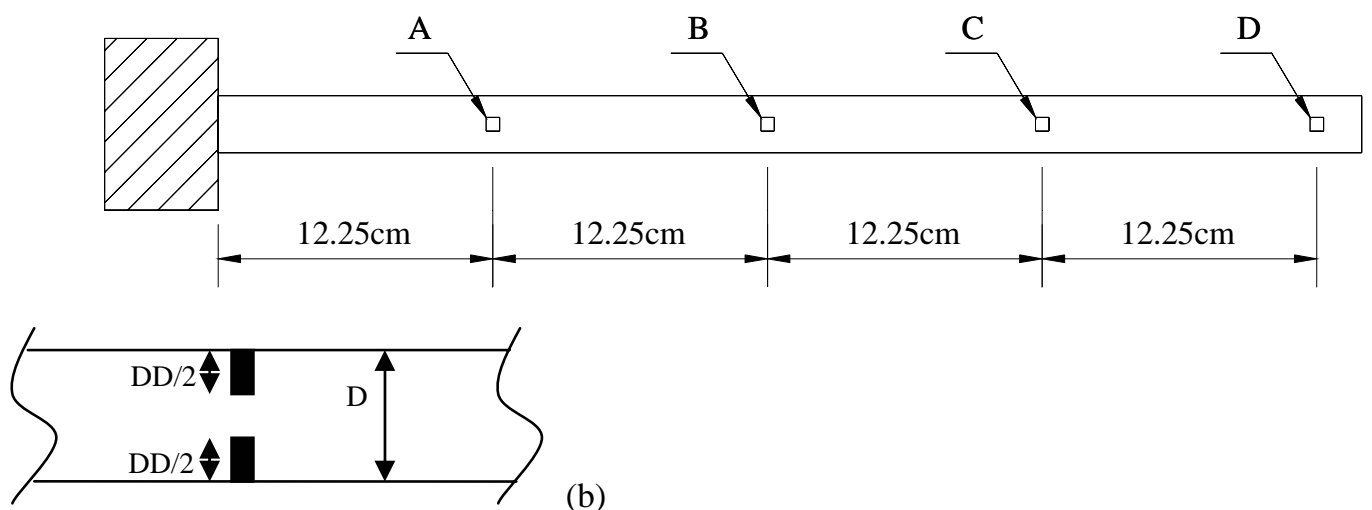

(a)

FIGURE 3

SiMULATED BEAM ELEMENT: (A) PLAN VIEW, POINTS OF INSTRUMENTATION; (B) PLAN VIEW, INTRODUCTION OF DAMAGE

\begin{tabular}{lcccc}
\hline Section Damage (DD) & $5 \% \mathrm{D}$ & $10 \% \mathrm{D}$ & $15 \% \mathrm{D}$ & $20 \% \mathrm{D}$ \\
\hline$h$ (strain-driven ARMA method) & 1.08 & $\mathbf{1 . 1 8}$ & $\mathbf{1 . 3 7}$ & $\mathbf{1 . 6 5}$ \\
$h$ (LANL method) & 1.15 & 1.21 & $\mathbf{1 . 3 5}$ & $\mathbf{1 . 5 8}$ \\
\hline Note: Numbers in bold indicate detected damage. & & & \\
\hline
\end{tabular}

TABLE 1

Performance of System IDENTIFication SChemes on Simulated BeAm Data AT L/2

In terms of localization capabilities, the proposed method was applied to strain and acceleration outputs at all four locations along the simulated damaged beam for five different damage scenarios, summarized in Table 2. It was speculated in [10] that the largest values of the damage metric would be observed near the damage location. In this case, damage is again imparted at $\mathrm{L} / 2$. The scheme detects damage from measurements at this location and at $\mathrm{L} / 4$ for all the damage cases considered, without false positives. Damage levels were slightly elevated at $\mathrm{L} / 4$ in comparison to those at $\mathrm{L} / 2$. This would indicate that localization capabilities can be somewhat demonstrated, though they were not largest at the exact location of damage. Future work will look at denser sensor arrays and the imparting of damage at non-measurement locations to examine further localization capabilities. Note that damage is scarcely detected in the second half of the beam. Lack of any detection capabilities at 3L/4 is actually due the presence of a 2nd mode node at that location. 


\begin{tabular}{|c|c|c|c|c|c|}
\hline Section Damage (DD): & $0 \%$ & $10 \%$ & $15 \%$ & $20 \%$ & $25 \%$ \\
\hline \multicolumn{6}{|c|}{ Location = L/4 (threshold = 1.13) } \\
\hline & 1.07 & 1.20 & 1.28 & 1.38 & 1.51 \\
\hline \multicolumn{6}{|c|}{ Location = L/2 (threshold = 1.11) } \\
\hline & 1.05 & 1.18 & 1.23 & 1.29 & 1.38 \\
\hline \multicolumn{6}{|c|}{ Location $=3 \mathrm{~L} / 4$ (threshold $=1.28$ ) } \\
\hline & 1.02 & 0.96 & 0.98 & 0.99 & 0.99 \\
\hline \multicolumn{6}{|c|}{ Location = L (threshold $=1.19$ ) } \\
\hline & 1.05 & 1.10 & 1.13 & 1.19 & 1.23 \\
\hline
\end{tabular}

TABLE 2

Damage Metric $h$ Demonstrating Localization capabilities of Proposed Damage Detection SCHEME

Reduction of Reference Pool. One of the major drawbacks of the LANL scheme is that it requires the user to establish a large reference pool of data from the bridge in its undamaged or reference state under the entire spectrum of operational and environmental conditions. If the reference pool is not truly comprehensive, the damage detection scheme will falter. Thus the memory required to store the reference pool and the computational power required to identify an appropriate reference state can be quite daunting for an actual bridge. In fact, as Lynch et al. [8] worked to embed the original LANL scheme into their wireless network, they were forced to resort to a remote data server to store the reference pool and perform all computations involving it. Unfortunately, this approach requires additional wireless communications, which are power intensive; however, if the reference pool could be somehow reduced, then a remote server may not be necessary. Furthermore, reduction of the reference pool to include only specific service conditions would also aid in system identification by providing a controlled input to the system, without the need for additional intrusive and expensive excitation equipment. Thus, the authors propose the use of RINAS to achieve this end.

Excitation by traffic is generally viewed as a random ambient process, assuming we have no measured input. Thus blind system identification approaches using statistical pattern recognition [11] were proposed to differentiate meaningful changes in the system from those associated with the variable service and environmental conditions. The use of controlled excitations provides knowledge of input and thus negates the need for such reference databases. The authors propose a compromise: activate the sensor network on a scheduled basis, e.g., weekly or monthly, and only when a particular input condition exists, e.g., only under the passage of an isolated semi-trailer, which may for example occur overnight. In this way, the input to the system is reduced to a specific loading scenario as opposed to a random distribution of vehicles at varying locations and velocities for a two fold benefit: dramatic reduction of the reference pool size and improved identification of damage since the inputs are more strictly controlled and easily encompassed by the reference pool. This type of restricted activation can be achieved by installing any number of vehicle classification technologies, including weigh-in-motion devices, inductive loop detectors, fiber optic sensors and wireless magnetic sensors. However, for the purposes of this study, video imaging technologies will be employed, as described in the subsequent section. Meanwhile, the environmental state can be more accurately identified by integrating outputs from on-board temperature and humidity sensors into the reference database as an additional parameter used in the identification of 
an appropriate reference state. Thus, through RINAS and the provision for environmental sensors, the reference pool can be vastly reduced, a suitable reference record can be identified with greater certainty, and the reliability of the ensuing damage identification process can be significantly improved, all without the need for costly communications with a remote server.

\section{Objective 3: Hardware Platform Design}

Though there have been a number of proprietary wireless platforms, the open hardware/software platform of the MICA Motes developed at the University of California at Berkeley is adopted here [6]. This platform's capabilities have already been demonstrated for a variety of applications. In this study, this platform, which has largely been used for vibration measurements by accelerometers, will be adapted to incorporate multiple strain measurements to form a $\mu$-web. It should be noted that recent proprietary designs by Lynch et al. [8] have also decoupled the sensor board from the wireless platform to create a similar wireless datalogger that can be interfaced with a variety of sensors. The intent of this effort is to first use a commercially available platform for the aforementioned reasons in a tethered format. As this $\mu$-web occupies a small region of the structure, the tethers incur minimal installation effort and cost. Thus, while the long term vision of a multi-scale wireless network concept would have a holistic wireless approach, the use of strain gages ( $\mu$-nodes) tethered to an m-node is viewed as an obvious first step in this network's evolution. Again, low power and low cost are central driving forces in selecting hardware components in this application and will play a central role in any selections. Note that the field of embedded electronics is rapidly expanding with new, more capable and lower-cost devices emerging almost weekly. So the description below should be viewed in this light, and it is a fact that there will probably be changes by the time these devices are implemented in full-scale.

Hardware Overview. The design features a NEMA4X-grade enclosure containing a remote bridge monitoring unit (RBMU). The RBMU consists of a sensor board, a MICA2 or MICAZ, which will be referred to as the MICA*, mote from Crossbow and a battery pack for powering the sensor board and MICA*. The MICA* will serve as the radio and handle any necessary ad-hoc routing algorithms. The Sensor Board will consist of signal conditioning electronics for the strain gages, the m-node's accelerometer, temperature and humidity sensors and a low cost processor with an external flash and SRAM to perform data logging and digital signal processing tasks. This processor will be enabled by the MICA* mote to perform data logging and signal processing tasks as necessary in order to maximize battery life. This low-cost processor can currently be from a broad range of families such as the ATMEGA128L, which was used by Wang et al. [3], or the more powerful 16 or 32 bit family of controllers, e.g., from the Renesas M16C family of controllers that provide ultra-low power consumption with high-speed processing capabilities.

Sensor Details. Strains will be measured by a three-wire quarter bridge circuit, whose outputs will be amplified and filtered by a differential amplifier, INA338, to render a fullscale signal to the MSP430F1611 12-bit A/D. Temperature compensation will be available from an on-board temperature sensor so that static-type gages can be employed to detect absolute stress levels. Given the trade-offs in accelerometer noise properties and cost, prototypes will consider a number of commercially available accelerometers, 
including those by Applied MEMS, the ADXL202E and ADXL213 by Analog Devices, and ICP Sensors by Honeywell. These will be evaluated within in subsequent validation stages to see whether the proposed data fusion will offset reduced sensor accuracy. Roughly considering the capabilities of these various units, sensing bandwidth will be limited to $20 \mathrm{~Hz}$ by analog AA Filters to minimize noise. In these configurations, the system will likely sample dynamic responses at up to $100 \mathrm{~Hz}$ with strains and accelerations within the limits roughly specified in Table 3.

\begin{tabular}{llll}
\hline Quantity & Resolution & Frequency Range & Operational Range \\
\hline Strain & $\pm 0.25 \mu \varepsilon$ & $0-50 \mathrm{~Hz}$ & $\pm 1000 \mu \varepsilon$ \\
Acceleration & $0.2-0.4 \mathrm{mg}$ & $\sim 0-20 \mathrm{~Hz}$ & $\pm 1200 \mathrm{mg}$ \\
\hline
\end{tabular}

TABLE 3

STRAIN \& ACCELERATION SENSING LIMITS

RINAS Sensor Interface. As discussed previously, RINAS is proposed to reduce input uncertainty in the system identification process by triggering the system only under the passage of isolated, large payload vehicles such as semis, as identified by an imaging system [13]. The authors will evaluate several commercially-available, low-cost options including the Wireless-G Network Camera by Hawking Industries (HNC230G), which can wirelessly transmit 30 frames per second to the Stargate M-node via $802.11 \mathrm{~b} / \mathrm{g}$. At the M-node, the frames will be processed in real-time to estimate vehicle weight and speed and make a decision whether to trigger the network, in accordance with a predefined schedule. The camera will be placed sufficiently upfield of the bridge to allow for transmission of image data and decision-making before the vehicle reaches the instrumented segment of the bridge. Though the authors have been working with this unit, other options, e.g., by Panasonic, Motorola and Intel, will be evaluated for the prototyping stage. Once the initial concept is verified using one of these low-cost solutions, a more robust imaging system, equipped for the environmental demands of a permanent installation, will be developed.

Initial Proof of Concept. Various prototypes in the literature have varied computational capabilities (processor selection) and internal RAM resources. Since the assembly of hardware is relatively straightforward, this section now addresses the larger concern of providing sufficient though not excessive computational capabilities to execute the scheme. Frankly, though larger processors can be used, they consume more power and were thus not considered options in this study. In actuality, the critical issue generally reduces to available internal RAM, as writing to the flash memory is costly, in terms of power. The proposed platform relies only upon its $10 \mathrm{~KB}$ of internal RAM and will use the proposed heterogeneous system identification approach to permit a trade off in model orders to eliminate writing to the flash memory. The most critical aspect of the implementation of the algorithms on motes is definitely the matrix inversion operation necessary for the solution of the Yule-Walker equations. The matrix to be inverted contains estimates of the discrete time history autocorrelation function and thus is a Toeplitz matrix, which permits relatively efficient inversion. A total of about $2 p^{3}$ divisions and multiplications are needed. The algorithm has been successfully implemented on only $4 \mathrm{~KB}$ of internal RAM for $p=8$, and it was observed that this was sufficient for not only this order, but also model orders up to 15, as demonstrated in Table 
4. As shown previously, the proposed system identification approach can detect damage for such relatively modest model orders. Also note that an additional $6 \mathrm{~KB}$ of RAM will be available in the proposed hardware design. Note that the limited accuracy of the 8-bit microcontroller causes some precision loss on the estimated parameters.

\begin{tabular}{cccccc}
\hline & $\mathrm{p}=8$ & & & $\mathrm{p}=15$ & \\
Mote & MATLAB & Error & Mote & MATLAB & Error \\
\hline 0.54432 & 0.56003 & $3 \%$ & -6.2972 & -6.4773 & $3 \%$ \\
-4.22822 & -4.24463 & $0 \%$ & 2.5644 & 2.7011 & $5 \%$ \\
2.65337 & 2.55412 & $4 \%$ & 5.9705 & 6.0271 & $1 \%$ \\
1.40866 & 1.55892 & $10 \%$ & -3.4564 & -3.5836 & $4 \%$ \\
2.36874 & 2.30359 & $3 \%$ & -2.2691 & -2.2268 & $2 \%$ \\
3.3254 & 3.39063 & $2 \%$ & 7.6161 & 7.7071 & $1 \%$ \\
-3.72192 & -3.73871 & $0 \%$ & -3.6788 & -3.7725 & $2 \%$ \\
-1.85879 & -1.89296 & $2 \%$ & -2.6436 & -2.6776 & $1 \%$ \\
& & & 7.3872 & 7.5693 & $2 \%$ \\
& & & -2.8217 & -2.9575 & $5 \%$ \\
& & & -2.9586 & -2.9679 & $0 \%$ \\
& & & 6.2588 & 6.4115 & $2 \%$ \\
& & & 1.7927 & 1.7287 & $4 \%$ \\
\hline
\end{tabular}

TABLE 4

AR CoefFicients Demonstrating THe Performance of EMBEDDED ALGORITHM ON WireLESS PLATFORM WITH 4 KB OF RAM

\section{ObJective 4: VALidation Stages}

At this stage, preliminary validations of the scheme have been concerned with simulated data. Validation is now being undertaken by the authors in three stages, discussed below.

\section{Stage 1: Integrated Simulation}

In the context of the design and validation of the decentralized system identification scheme, this first stage validation must be preceded by a verification of the RINAS scheme and of the damage detection accuracy using full-scale data. Through a partnership with the Indiana Department of Transportation (INDOT), the authors will collect in-situ response data from an in-service post-tensioned reinforced concrete bridge frequently traveled by large payload semi-trailers, referred to herein as the pilot bridge. Response, environmental and video imaging data will be collected using a traditional wired data acquisition system under a variety of service conditions. The full-scale data set will enable the authors to verify the improvement of system identification accuracy when the analysis is conducted using only those records characterized by the passage of isolated, large payload vehicles, consistent with the RINAS scheme, in comparison to the random traffic conditions that may be encountered without RINAS.

Subsequently, verification of the broader system identification scheme will be conducted using a finite element model of this pilot bridge, calibrated to the full-scale data set, assumed to represent the undamaged state, followed by the subsequent introduction of damage, with varying severity and location, in the numerical model. Once the system identification approach is independently validated using this pilot bridge model, an integrated simulator will be developed in MATLAB, with the damaged pilot 
bridge model providing the structural simulator, which is then interfaced with Prowler, a MATLAB-based sensor networks simulator developed at Vanderbilt University. Prowler is a probabilistic network simulator capable of emulating wireless distributed systems, from the application to the physical communication layer. In particular, it incorporates realistic wireless channel models and thus provides accurate results.

\section{Stage 2: Bench Scale Experimentation}

In many investigations in the literature, progressive damage is simulated in stages, beginning with the undamaged baseline structure, which is benchmarked, followed by successive damage accompanied by re-testing. As the proposed multi-scale network will be triggered by RINAS, the detection of damage will always be progressive. Thus for simplicity, the initial multi-scale network concept will be experimentally validated on a suite of models emulating multispan bridges with varying damage levels introduced by cyclic loading so as to represent realistic damage mechanisms. This suite can then be used repeatably in each of the following phases.

Three phases of bench scale experimentation are proposed: independent verification of the detection scheme (Phase A), independent verification of the wireless hardware (Phase B) and the integrated system testing (Phase C). In Phase A, the detection scheme will be validated using sensing elements wired to a centralized data acquisition unit. Here each sensor's output will be independently processed by the proposed identification scheme to mimic the on-board operations of the motes, allowing the sensitivity of the identification scheme to be assessed for a suite of damage levels, devoid of any influence of the wireless platform. Phase B will utilize a dynamically-excited undamaged beam to evaluate the performance of various accelerometer prototypes. Once the final hardware design is verified, this phase of testing will continue to verify the platform's ability to accurately perform the data fusion, as well as the functionality of communications, routing and operation of the network hierarchy. Finally, in Phase $\mathrm{C}$, the wireless network will be deployed on the specimens to validate integrated capabilities.

\section{Stage 3: Full-Scale Deployment}

While the integrated simulator (Stage 1) and bench scale verifications (Stage 2) will establish the efficacy of the network concept, its supporting hardware and the computational algorithms, full-scale verifications are necessary to develop the RINAS interfacing and establish the overall proof-of-concept. Prototyping and development of the RINAS hardware, its interface with the wireless sensor network, and triggering mechanism will be conducted using roadways on the campus of the University of Notre Dame, providing a relatively controlled environment.

Following the field verifications of the RINAS system, the final phase of this program will include a temporary deployment of the wireless sensor network with RINAS on the pilot bridge in parallel with a traditional wired system. As controlled damage cannot be introduced, this exercise is intended to evaluate the field performance of the hardware.

\section{SUMMARY}

As our country now grapples with strategies to assess and repair its expansive network of aging roadways and bridges, the use of wireless SHM can provide the quantitative and continuous metric of structural health that is so needed. Recognizing the limitations 
imposed by computational limitations and finite power supply, inherent to any practical wireless network, this study recasts the concept of network architecture to a multi-scale format with data fusion and spatially distributed, heterogeneous sensing and a restricted input network activation scheme. Preliminary results on simulated data indicate that such a network will offer improved reliability of damage detection and localization through the fusion of data from different, spatially distributed sensors, reducing the size of reference databases and requisite model orders to relieve computational burden and extend network lifetime. Power efficiency is further enhanced through the adoption of a multi-scale network architecture that utilizes localized processing and activates only those sensors in the vicinity of damage. The authors will next begin to engage in the aforementioned experimental validation stages to provide further verification of the multi-scale network concept.

\section{ACKNOWLEDGEMENTS}

The authors wish to acknowledge the financial support of the University of Notre Dame and the efforts of Nicholas Kottenstette, Daniele Puccinelli, Su Su and Brian Stein.

\section{REFERENCES}

[1] Straser, E. G., Kiremidjian, A. S., “A Modular, Wireless Damage Monitoring System for Structures”, John A. Blume Earthquake Engineering Center, Department of Civil and Environmental Engineering, Stanford University, Report No. 128, 1998.

[2] Lynch, J. P., Sundararajan, A., Law, K. H., Kiremidjian, A. S., Kenny, T. and Carryer, E., "Embedment of Structural Monitoring Algorithms in a Wireless Sensing Unit”, Structural Engineering and Mechanics, 15, March 2003, 285-297.

[3] Wang, Y., Lynch, J.P., Law, K.H., "A Wireless Structural Health Monitoring System with Multithreaded Sensing Devices: Design and Validation”, Structure and Infrastructure, in press, 2005.

[4] Xu, N., et al., “A Wireless Sensor Network for Structural Monitoring”, Proceedings of ACM SenSys'04, Nov. 2004.

[5] Hill, J., et al., "System Architecture Directions for Networked Sensors", Proceedings of International Conference on Architectural Support for Programming Languages and Operating Systems (ASPLOS), Nov. 2000.

[6] Kijewski-Correa, T., Haenggi, M., Antsaklis, P., "Multi-Scale Wireless Sensor Networks for Structural Health Monitoring”, Proceedings of SHM-II'05, Nov. 16-18, 2005.

[7] Law, S., Li, X., Zhu, X., Chan, S., "Structural Damage Detection from Wavelet Packet Sensitivity", Engineering Structures, 27, 2005, 1339-1348.

[8] Lynch, J.P., Sundararajan, A., Law, K.H., Kiremidjian, A.S., Carryer, E., "Embedding Damage Detection Algorithms in a Wireless Sensing Unit for Operational Power Efficiency”, Smart Materials and Structures, 13, 2004, 800-810.

[9] Doebling, S.W., Farrar, C.R., The State of the Art in Structural Identification of Constructed Facilities, ASCE Committee on Structural Identification of Constructed Facilities, 1999.

[10] Caffrey, J., et al., "Networked Sensing for Structural Health Monitoring”, Proceedings of 4th International Workshop on Structural Control, June 2004.

[11] Sohn, H., Farrar, C.R., "Damage Diagnosis Using Time Series Analysis of Vibration Signals”, Smart Materials and Structures, 10, 2001, 446-451.

[12] Fasel, T., Sohn, H., Park, G., Farrar, C., "Active Sensing Using Impedance-Based ARX Models and Extreme Value Statistics for Damage Detection”, Earthquake Engineering and Structural Dynamics, 34, 2005, 763-785.

[13] Elgamal, A., et al., “A Framework for Monitoring Bridges and Civil Infrastructure”, Proceedings of 3rd China-Japan-US Symposium on Structural Health Monitoring and Control, Oct. 2004. 